\title{
Costo-efectividad de un tratamiento antituberculoso alternativo: seguimiento a convivientes residenciales de los pacientes
}

\author{
Emmanuel Nieto, ${ }^{1}$ Lucelly López, ${ }^{2}$ Helena del Corral, ${ }^{2}$ \\ Diana Marín, ${ }^{1}$ Luz Dolly Lopera, ${ }^{1}$ Dione Benjumea, ${ }^{1}$ Fernando Montes, ${ }^{3}$ \\ Gloria Molina ${ }^{1}$ y María Patricia Arbeláez ${ }^{1}$
}

Forma de citar Nieto E, López L, del Corral H, Marín D, Lopera LD, Benjumea D, et al. Costo-efectividad de un tratamiento antituberculoso alternativo: seguimiento a convivientes residenciales de los pacientes. Rev Panam Salud Publica. 2012;32(3):178-84.

RESUMEN Objetivo. Estimar la relación de costo-efectividad del tratamiento de corta duración bajo observación directa (DOTS), comparándolo con una variación de dicho tratamiento, que incluye un mayor seguimiento a los convivientes residenciales de los pacientes (DOTS-R) para el tratamiento de tuberculosis (TB).

Métodos. Tomando una perspectiva social que incluye los costos para las instituciones de salud, para los pacientes y sus familiares, y para otras entidades que contribuyen a hacer efectiva la operación del programa, se evaluaron los costos incurridos con cada una de las dos estrategias y se estimaron razones costo-efectividad adoptando las medidas de efecto usadas por los programas de control. La estimación de los costos de cada una de las dos estrategias incluye los correspondientes a las instituciones de salud que administran el tratamiento, los pacientes y sus familiares, y los de la secretaría de salud que gestiona los programas de salud pública a nivel municipal. Con base en estos costos y el número de casos curados y tratamientos terminados como medidas de resultado de cada una de las estrategias evaluadas, se calcularon las razones costo-efectividad y costo incremental.

Resultados. El DOTS-R se halló más costo-efectivo para lograr tratamientos exitosos que el DOTS. El DOTS-R registró costos de entre US\$1 122,4 y US\$1 152,7 por caso curado, comparados con valores de entre US\$ 1 137,0 y US\$1 494,3 correspondientes al DOTS. La proporción de casos tratados con éxito fue mayor con DOTS-R que con DOTS.

Conclusiones. El DOTS-R es una alternativa costo-efectiva promisoria para mejorar el control de la TB en sitios endémicos. Se recomienda a las autoridades del sector salud incorporar en su gestión institucional del programa contra la TB, acciones de seguimiento de los convivientes de pacientes, con la participación del personal de salud y los recursos físicos y financieros que apoyan actualmente dicho programa.

Palabras clave Tuberculosis pulmonar; análisis costo-eficiencia; financiación de la salud; Colombia.

La tuberculosis (TB) es una infección causada por Mycobacterium tuberculosis,

\footnotetext{
1 Facultad Nacional de Salud Pública, Universidad de Antioquia, Medellín, Colombia. La correspondencia se debe dirigir a María Patricia Arbeláez. Correo electrónico: mpam@saludpublica.udea.edu.co

2 Escuela de Microbiología, Universidad de Antioquia, Medellín, Colombia.

3 Secretaría de Salud de Medellín, Colombia.
}

generalmente afecta a los pulmones, se transmite de persona a persona a través del aire, es curable y se puede prevenir. Después del sida, es la segunda causa mundial de mortalidad provocada por un agente infeccioso. En 2010, 8,8 millones de personas enfermaron de tuberculosis $y$ 1,4 millones murieron por esta causa (1).
Desde 1995, gracias a la estrategias DOTS $^{4}$ y Alto a la Tuberculosis, recomendadas por la Organización Mundial de la Salud (OMS), la afección se ha tratado con buenos resultados en más de 46

\footnotetext{
4 Siglas en inglés de "tratamiento de corta duración bajo observación directa".
} 
millones de personas y se calcula que se habrían salvado alrededor de 7 millones de vidas (1-3).

Sin embargo, y a pesar de que esta estrategia ha mejorado significativamente la situación de la TB en la Región de las Américas, los programas para su control efectivo aún tropiezan con serios obstáculos vinculados al debilitamiento de las acciones en salud pública y el deterioro social que se desprenden de la racionalidad de mercado imperante desde los años noventa (4). A su vez, la TB es una rémora para el desarrollo socioeconómico de los países, porque impone importantes costos no solo al sistema de salud sino también a los pacientes o sus familias (5).

Varios estudios realizados en diferentes países han evaluado la efectividad - y documentado las ventajas - del DOTS en comparación con otras estrategias de tratamiento antituberculoso. Algunos han demostrado que también reduce sustancialmente los gastos en salud vinculados al tratamiento de la TB (6-12). En el marco de las recomendaciones de la OMS, se han publicado varios estudios sobre la costo-efectividad de la estrategia DOTS, realizados en Brasil, India y en algunos países africanos. Más recientemente, las investigaciones han optado por evaluar y comparar al DOTS no solo con respecto a otros tipos de tratamientos, sino también en relación a las distintas opciones que proporciona, hallándose evidencias a favor de una aplicación que incluya en su ejecución la participación comunitaria y la de familiares de los pacientes $(7,13-24)$.

Dado este contexto, el objetivo del presente estudio fue precisamente estimar la relación de costo-efectividad del DOTS, comparándolo con una variación de dicho tratamiento que incluye un mayor seguimiento a los convivientes residenciales (DOTS-R) en la ciudad de Medellín (Colombia).

\section{MATERIALES Y MÉTODOS}

Se realizó una evaluación económica tipo costo-efectividad que permitió comparar los costos y resultados de dos estrategias de implementación del DOTS. La población de estudio incluyó casos nuevos de TB pulmonar confirmada por baciloscopia diagnosticados en la ciudad de Medellín. Se constituyeron dos escenarios de comparación: uno simultáneo en 2006 y otro antes-después.

\section{Población de estudio}

En 2006 se comparó un grupo de 371 pacientes diagnosticados por el programa de control y tratados con la estrategia DOTS, con otro de 164 pacientes, también diagnosticados durante ese año, pertenecientes a una muestra de 366 pacientes a quienes - entre 2005 y 2008 - se les había efectuado un seguimiento adicional que involucraba a sus grupos familiares y residenciales. Esta variación de la estrategia DOTS (denominada DOTS-R) surgió en el marco de un proyecto de investigación enfocado en una cohorte de convivientes residenciales de pacientes con TB pulmonar (25). La comparación antes-después se hizo entonces entre el grupo de pacientes intervenido con DOTS-R $(n=366) \mathrm{y}$ los 642 pacientes diagnosticados con TB pulmonar en 2009 —en Medellín-que no recibieron seguimiento.

\section{Intervenciones evaluadas}

La intervención con el DOTS-R consistió en agregar al DOTS una serie de actividades llevadas a cabo durante los dos años posteriores al diagnóstico, que incluyeron i) visitas residenciales cada seis meses (suplementarias a las contempladas por el DOTS) para estudio de contactos; ii) llamadas telefónicas de seguimiento cada tres meses, intercaladas entre las visitas, y iii) disponibilidad de personal capacitado para consulta y notificación de síntomas por vía telefónica durante los días hábiles de la semana. Todas estas actividades incluyeron, de manera sistemática, acciones de educación en salud. La intervención DOTS, por su parte, consistió en la supervisión directa del tratamiento en el centro de salud durante seis meses, y una visita domiciliaria durante los primeros días posteriores al diagnóstico para el estudio de los contactos (26).

\section{Análisis de efectividad}

Los desenlaces eventuales de los pacientes con TB pulmonar que interesaban para la evaluación de efectividad fueron curación, tratamiento terminado, fracaso del tratamiento, abandono del tratamiento y muerte. Los pacientes transferidos o sin datos completos fueron excluidos de los análisis de efectividad.

Para el cálculo de efectividad se compararon los desenlaces del grupo intervenido con el DOTS-R con los del grupo intervenido con el DOTS. En el caso de la comparación simultánea de 2006, las fuentes de información para el grupo no intervenido fueron los datos agregados de los registros proporcionados por la Secretaría de Salud de Medellín, exceptuando la información de los 366 pacientes que fueron intervenidos con DOTS-R. Para este último grupo, se utilizó la información consignada en la tarjeta individual de tratamiento de cada paciente. Para la comparación antes-después, el grupo intervenido con DOTS-R se contrastó con la información proveniente de las fichas individuales de tratamiento de 642 pacientes de TB pulmonar diagnosticados con baciloscopia durante el año 2009.

La efectividad fue calculada de dos maneras: comparando desenlaces curados contra el resto de posibles desenlaces, e introduciendo una categoría denominada "éxitos", que incluía pacientes curados y con tratamiento terminado. Para esta última categoría, el grupo de comparación incluyó a los pacientes con abandono, fracaso y fallecidos agrupados como "otros".

\section{Análisis de costos}

El costeo del DOTS y el DOTS-R se efectuó con una perspectiva social; los costos se llevaron a dólares estadounidenses del año 2010. La perspectiva social exigió abordar los costos no solo del tratamiento que dispensan las instituciones prestadoras de los servicios, sino también los correspondientes a los pacientes y sus familiares, así como los de la gestión del programa por parte de la Secretaría de Salud. A estos tres ámbitos de costeo, se les agregó el componente de seguimiento de los convivientes incorporado en la estrategia DOTS-R. Cada uno de los anteriores ámbitos se abordó con micro-costeo, aunque en algunos casos fue preciso utilizar el costeo de arriba-abajo.

La información requerida para valorar los recursos utilizados por las instituciones de salud provino de una muestra de 16 de estas instituciones que operan en la ciudad de Medellín: 6 públicas y 10 privadas. Para estos efectos se diseñó y administró un formulario estandarizado, el cual permitió consolidar la información relacionada con recursos humanos, exámenes y medicamentos, suministros, servicios generales, equipamiento y edificios. La valoración monetaria de estos recursos se hizo con base en precios 
de mercado e información de estas 16 instituciones.

Siguiendo la Guía metodológica de la OMS y las recomendaciones provenientes de la literatura especializada $(6,18$, 19), se optó por valorar los tres grandes tipos de costos en que suelen incurrir los pacientes y sus familiares en la búsqueda del tratamiento contra la TB: i) gastos de viajes; ii) gastos de bolsillo para pago de servicios de consulta externa, medicamentos y demás, y iii) pérdidas de productividad.

La información requerida para la estimación de los costos que afectan a los pacientes o a sus familiares se obtuvo mediante la aplicación de un cuestionario estandarizado a una muestra representativa de 150 pacientes. La valoración de los tiempos de viaje y de espera en los servicios de salud se basó en los informes de ingresos laborales de los propios pacientes y de sus familiares. En ausencia de esta información, se utilizó el promedio de los ingresos laborales reportados en la Encuesta de Calidad de Vida de Medellín (27).

Para la estimación de los costos del manejo del programa de control de TB por la Secretaría de Salud, se contactó al personal encargado del programa para la información correspondiente a recursos de personal, servicios generales, equipamiento, instalaciones y contratación externa. El equipamiento y las instalaciones se valoraron con base en precios de mercado. Los demás recursos se valoraron con la información suministrada por la propia Secretaría de Salud.

Para el desarrollo de las actividades de seguimiento a los convivientes, se identificaron requerimientos de recursos humanos, exámenes de laboratorio, suministros, servicios generales, equipamientos, instalaciones y transporte, los cuales fueron valorados con base en los registros contables del proyecto de investigación que dio lugar al grupo intervenido con DOTS-R. El seguimiento domiciliario del tratamiento anti-TB generó también pérdidas de productividad en pacientes y familiares, que fueron valoradas con base en los reportes de ingresos laborales de los 150 pacientes encuestados.

\section{Análisis de costo-efectividad}

Los efectos de las alternativas evaluadas fueron expresados como porcentaje de casos curados después de seis meses de tratamiento contra la $\mathrm{TB}$, o como porcentaje de casos tratados con éxito (curados y con tratamiento terminado).

La medida resumen utilizada para los análisis de costo-efectividad fue la razón costo-efectividad, expresada en términos medios (costos/efectos) o incrementales (costos de A - costos de B)/(efectos de A - efectos de B). Los resultados de estas medidas fueron objeto de análisis de sensibilidad.

\section{Análisis de sensibilidad}

Dado que en el presente trabajo la valoración de los costos asumió un horizonte de tiempo de seis meses, los costos no son sensibles a tasa de descuento anual. Además, todas las mediciones de costos se llevaron a dólares estadounidenses del año 2010 y la intervención evaluada no era totalmente diferente, sino una adición a la estrategia convencional. En consecuencia, el análisis de sensibilidad se enfocó en examinar el impacto sobre los resultados que podría tener el hecho de usar dos grupos de comparación diferentes: unos de un mismo año (2006) y otros de dos (antesdespués [2006 vs 2009]).

\section{Análisis estadístico}

Se incluyó una comparación sociodemográfica de los grupos a partir de la estimación de proporciones y medias con desviación estándar. Se utilizaron las pruebas $Z$ y $U$ de Mann-Whitney para establecer diferencias significativas utilizando la corrección de Bonferroni. Para la comparación de las medidas de efectividad entre el DOTS-R y el DOTS, se calcularon las proporciones, la magnitud del efecto a partir de la diferencia de esas proporciones y la prueba $Z$ para su significación estadística, estableciendo un valor $P$ de significación estadística menor a 0,05. Para la comparación de los costos, se estimaron los costos medios y la frecuencia relativa de cada tipo de costo.

Se utilizó una regresión binomial con función de enlace logístico y varianza binomial para ajustar la efectividad por edad, sexo y régimen de afiliación en salud; en este caso se reportó el riesgo relativo (RR), ajustado y no ajustado, de presentar un resultado no exitoso (desfavorable) en el tratamiento, y la efectividad del DOTS-R frente al DOTS se interpretó como (1 - RR) × 100 (28).
Para todas las estimaciones se calcularon intervalos de confianza del 95\% (IC95\%). La base de datos fue administrada en Access y el procesamiento se realizó en Stata 10.1 y en PASW 17.0

\section{Aspectos éticos}

Tanto para la información captada de los pacientes intervenidos con estrategia DOTS-R, como para la que aportaron los pacientes sobre costos propios y de familiares, se obtuvo consentimiento informado individual. El estudio contó con la revisión y aprobación del comité de ética de la Facultad Nacional de Salud Pública de la Universidad de Antioquia y el del Instituto Metropolitano de Salud de Medellín.

\section{RESULTADOS}

El grupo de pacientes que recibieron DOTS-R fue comparable en la mayoría de las variables a los pacientes que recibieron DOTS (cuadro 1). Se encontraron diferencias por régimen de afiliación en salud: $44 \%$ de los pacientes del DOTS-R en régimen contributivo frente a $30,7 \%$ en el DOTS.

La efectividad del DOTS-R fue superior al DOTS, tanto en la comparación de las cohortes del mismo año (cuadro 2), como en los períodos diferentes (cuadro 3). Al comparar los tratamientos exitosos antes y después, se halló que 85,5\% de los pacientes del DOTS-R tuvieron un tratamiento exitoso, mientras que en los pacientes del DOTS la proporción fue de $66,1 \%$ (sumatoria de pacientes curados y tratamientos terminados). En cuanto al abandono del tratamiento, las proporciones fueron $4,7 \%$ con DOTS-R y $28,2 \%$ con DOTS $(P<0,001)$ (cuadro 3 ).

Al ajustar la efectividad del DOTS$R$ según las variables en las que se encontraron diferencias con el DOTS, se observó una menor probabilidad de obtener un resultado desfavorable en el grupo con DOTS-R (RR: 0,514; 0,39-0,68). Asimismo, se halló que los hombres, y quienes tenían un régimen de afiliación en salud diferente al contributivo, tuvieron mayor riesgo de no terminar exitosamente el tratamiento (cuadro 4).

En respuesta a la adopción de la perspectiva social en el análisis de los costos, en el cuadro 5 se pueden ver por separado los costos medios para las instituciones prestadoras de servicios de salud, 
CUADRO 1. Caracterización sociodemográfica de pacientes con tuberculosis pulmonar que recibieron tratamiento de corta duración bajo observación directa (DOTS) y DOTS residencial (DOTS-R) entre 2006 y 2009, Medellín, Colombia

\begin{tabular}{|c|c|c|c|c|c|c|c|}
\hline \multirow[b]{2}{*}{ Característica } & \multicolumn{2}{|c|}{ DOTS $(n=642)$} & \multicolumn{2}{|c|}{ DOTS-R $(n=366)$} & \multicolumn{2}{|c|}{ Total } & \multirow[b]{2}{*}{$P^{a}$} \\
\hline & No. & $\%$ & No. & $\%$ & No. & $\%$ & \\
\hline \multicolumn{8}{|l|}{ Sexo } \\
\hline Femenino & 249 & 38,80 & 159 & 43,40 & 408 & 40,50 & 0,1670 \\
\hline Masculino & 393 & 61,20 & 207 & 56,60 & 600 & 59,50 & 0,1670 \\
\hline \multicolumn{8}{|c|}{ Régimen de afiliación en salud } \\
\hline Contributivo & 197 & 30,70 & 161 & 44,00 & 358 & 35,50 & $<0,0001^{b}$ \\
\hline Subsidiado & 255 & 39,70 & 121 & 33,10 & 376 & 37,30 & 0,0419 \\
\hline Vinculado & 169 & 26,30 & 80 & 21,90 & 249 & 24,70 & 0,1323 \\
\hline No afiliado & 0 & 0,00 & 4 & 1,10 & 4 & 0,40 & $0,0329^{b}$ \\
\hline Otro & 14 & 2,20 & 0 & 0,00 & 14 & 1,40 & $0,0103^{b}$ \\
\hline Sin datos & 7 & 1,10 & 0 & 0,00 & 7 & 0,70 & 0,1073 \\
\hline \multicolumn{8}{|l|}{ Edad (años) } \\
\hline $15-24$ & 120 & 18,70 & 92 & 25,10 & 212 & 21,00 & 0,0196 \\
\hline $25-49$ & 325 & 50,60 & 182 & 49,70 & 507 & 50,30 & 0,8351 \\
\hline $50-64$ & 129 & 20,10 & 62 & 16,90 & 19 & 18,90 & 0,2522 \\
\hline$\geq 65$ & 68 & 10,60 & 30 & 8,20 & 98 & 9,70 & 0,2611 \\
\hline Promedio (años) ${ }^{c}$ & \multicolumn{2}{|c|}{41,29} & \multicolumn{2}{|c|}{38,75} & \multicolumn{2}{|c|}{40,37} & 0,0090 \\
\hline Desviación estándar & \multicolumn{2}{|c|}{16,21} & \multicolumn{2}{|c|}{16,28} & \multicolumn{2}{|c|}{16,27} & $N A^{d}$ \\
\hline
\end{tabular}

Fuente: Elaboración de los autores con base en datos de la Secretaría de Salud de Medellín (29).

a Según la prueba Z para comparación de proporciones. (El nivel de significación es distinto en cada variable, por la corrección de Bonferroni.)

be utilizó la corrección de Bonferroni y se encontraron diferencias significativas $(0,05 / 6=0,008)$.

Según la prueba $U$ de Mann-Whitney.

d NA: Datos no aplicables.

CUADRO 2. Desenlaces de pacientes con tuberculosis pulmonar que recibieron tratamiento de corta duración bajo observación directa (DOTS) y DOTS residencial (DOTS-R) en 2006, Medellín, Colombia

\begin{tabular}{|c|c|c|c|c|c|c|c|c|c|}
\hline \multirow[b]{2}{*}{ Desenlace } & \multicolumn{3}{|c|}{ DOTS $(n=371)$} & \multicolumn{3}{|c|}{ DOTS-R $(n=164)^{\mathrm{a}}$} & \multicolumn{3}{|c|}{ Total $(n=535)$} \\
\hline & No. & $\%$ & IC95\% ${ }^{b}$ & No. & $\%$ & IC95\% & No. & $\%$ & $\mathrm{IC} 95 \%$ \\
\hline Curación & 223 & 68,6 & $63,4-73,8$ & 125 & 76,2 & $69,4-83,0$ & 348 & 71,2 & $67,0-75,3$ \\
\hline Tratamiento terminado & 37 & 11,4 & $7,8-15,0$ & 19 & 11,6 & $6,4-16,8$ & 56 & 11,5 & $8,5-14,4$ \\
\hline Fracaso & 4 & 1,2 & $0,3-3,1$ & 5 & 3,0 & $1,0-7,0$ & 9 & 1,8 & $0,5-3,1$ \\
\hline Abandono & 47 & 14,5 & $10,5-18,4$ & 7 & 4,3 & $0,9-7,7$ & 54 & 11,0 & $8,2-3,9$ \\
\hline Muerte & 14 & 4,3 & $1,9-6,7$ & 8 & 4,9 & $1,3-8,5$ & 22 & 4,5 & $2,6-6,4$ \\
\hline Transferidos & 44 & $\mathrm{NA}^{\mathrm{C}}$ & NA & 0 & NA & NA & 44 & NA & NA \\
\hline Sin datos & 2 & NA & NA & 0 & NA & NA & 2 & NA & NA \\
\hline
\end{tabular}

Fuente: Elaboración de los autores con base en datos de la Secretaría de Salud de Medellín (29).

a Se trata de 164 casos diagnosticados en 2006, de una cohorte de 366 que recibió seguimiento entre 2005 y 2008.

b Intervalo de confianza de $95 \%$.

${ }^{c}$ NA: Datos no aplicables.

CUADRO 3. Desenlaces de pacientes con tuberculosis pulmonar que recibieron tratamiento de corta duración bajo observación directa (DOTS) y DOTS residencial (DOTS-R) en 2006 y 2009, Medellín, Colombia

\begin{tabular}{|c|c|c|c|c|c|c|c|c|c|}
\hline \multirow[b]{2}{*}{ Desenlace } & \multicolumn{3}{|c|}{ DOTS $(n=642)$} & \multicolumn{3}{|c|}{ DOTS-R $(n=366)$} & \multirow{2}{*}{$\begin{array}{l}\text { Diferencia } \\
(\%)\end{array}$} & \multirow[b]{2}{*}{ IC95\% } & \multirow[b]{2}{*}{$P^{\mathrm{b}}$} \\
\hline & No. & $\%$ & IC95\% ${ }^{a}$ & No. & $\%$ & IC95\% & & & \\
\hline Curación & 218 & 52,2 & $47,2-57,1$ & 270 & 74,2 & $69,5-78,8$ & 22,0 & $15,2-28,8$ & $<0,001$ \\
\hline \multicolumn{10}{|l|}{ Tratamiento } \\
\hline terminado & 58 & 13,9 & $10,4-17,3$ & 41 & 11,3 & $7,9-14,6$ & $-2,6$ & $-7,5-2,3$ & 0,3232 \\
\hline Fracaso & 5 & 1,2 & $0,4-2,8$ & 18 & 4,9 & $2,6-7,3$ & 3,7 & $1,0-6,5$ & 0,0039 \\
\hline Abandono & 118 & 28,2 & $23,8-32,7$ & 17 & 4,7 & $2,4-7,0$ & $-23,5$ & $-28,6-18,5$ & $<0,001$ \\
\hline Muerte & 19 & 4,5 & $2,4-6,7$ & 18 & 4,9 & $2,6-7,3$ & 0,4 & $-2,8-3,6$ & 0,9253 \\
\hline Transferidos & 57 & $N A^{c}$ & NA & 0 & NA & NA & NA & NA & NA \\
\hline Sin datos & 167 & NA & NA & 2 & NA & NA & NA & NA & NA \\
\hline
\end{tabular}

Fuente: Elaboración de los autores con base en datos de la Secretaría de Salud de Medellín (29).

a Intervalo de confianza de $95 \%$.

b Prueba Z para comparación de proporciones.

${ }^{c}$ NA: Datos no aplicables. para los pacientes con sus familiares y para la Secretaría de Salud Municipal. El componente de menor incidencia en la estructura de costos del DOTS y el DOTS-R correspondió a los costos del sistema de salud.

Los costos medios para los pacientes y familiares, que se derivan fundamentalmente de las pérdidas de productividad, representaron cerca de la mitad de los costos medios totales, constituyéndose en el rubro más costoso. En ambos programas estos costos representaron cerca de $15 \%$ del ingreso semestral de los pacientes, lo cual no deja de ser significativo si se tiene en cuenta que $68 \%$ de estas personas pertenecen al estrato socioeconómico bajo.

Los datos de las razones costoefectividad y costo incremental, presentados en el cuadro 6, muestran que para los pacientes diagnosticados en el mismo año, por cada caso de curación con el DOTS-R se incurrió en un costo de US\$ 1 122,4 —US\$ 14,6 menos que el costo por caso curado reportado con el DOTS. Con objeto de determinar si las razones estimadas anteriormente eran consistentes, se realizaron cálculos similares utilizando las medidas de efectividad obtenidas en la comparación de un antes y un después (cuadro 6). Estos datos demostraron que las diferencias en las razones costo-efectividad se amplían a favor del DOTS-R.

\section{DISCUSIÓN}

Los resultados del presente estudio permiten concluir que, en Medellín, es costo-efectivo incorporar al DOTS un componente de seguimiento a pacientes y sus convivientes residenciales. Pese a que los resultados iniciales de la evaluación económica muestran solo una leve diferencia en costo-efectividad a favor del DOTS-R, hay buenas razones para creer que estos hallazgos son robustos. El análisis de sensibilidad abordado para explorar el impacto de usar dos tipos de grupos de comparación (diferentes en el tiempo), muestra que esta conclusión se mantiene. En efecto, con base en el antes-después, el grupo tratado con el DOTS-R amplía sus diferenciales de tasas de curación y de razones de costo-efectividad con respecto al DOTS, generando una sensible reducción en los costos incrementales.

Dada la naturaleza de la intervención denominada DOTS-R, cabe pensar que 
CUADRO 4. Efectividad del tratamiento de corta duración bajo observación directa (DOTS) residencial (DOTS-R), frente al DOTS, ajustada por sexo, edad y régimen de afiliación en salud, en pacientes con tuberculosis pulmonar, 2006 y 2009, Medellín, Colombia

\begin{tabular}{|c|c|c|c|c|c|}
\hline $\begin{array}{l}\text { Resultado desfavorable } \\
\text { en tratamiento }\end{array}$ & $\begin{array}{c}\mathrm{RR}^{\mathrm{a}} \\
\text { no ajustado }\end{array}$ & $\begin{array}{c}\text { RR } \\
\text { ajustado }\end{array}$ & Error estándar & $P$ & IC95\% ${ }^{\mathrm{C}}$ \\
\hline DOTS-R & 0,77 & 0,514 & 0,073 & $<0,001$ & $0,39-0,68$ \\
\hline \multicolumn{6}{|l|}{ Edad (años) } \\
\hline $25-49$ & 1,10 & 1,114 & 0,242 & 0,621 & $0,73-1,71$ \\
\hline $50-64$ & 1,14 & 1,081 & 0,256 & 0,741 & $0,68-1,72$ \\
\hline \multicolumn{6}{|l|}{ Sexo } \\
\hline $\begin{array}{l}\text { Hombre } \\
\text { Réaimen }\end{array}$ & 1,27 & 1,935 & 0,295 & $<0,001$ & $1,44-2,61$ \\
\hline $\begin{array}{l}\text { Régimen de afiliación } \\
\text { Subsidiado }\end{array}$ & 1,86 & 1,596 & 0,292 & 0,011 & $1,11-2,28$ \\
\hline Vinculado & 3,13 & 2,532 & 0,441 & $<0,001$ & $1,80-3,56$ \\
\hline
\end{tabular}

Fuente: Elaboración de los autores con base en datos de la Secretaría de Salud de Medellín (29).

Nota: Las categorías de referencia son: DOTS, Edad $\leq 14$ años, Mujer y Régimen de afiliación en salud contributivo.

a RR: riesgo relativo.

b Riesgo relativo como razón entre la proporción del desenlace del grupo que recibió DOTS-R, en comparación con el que recibió DOTS, ajustado por edad, sexo y régimen de afiliación en salud, utilizando regresión binomial $(n=782)$.

c Intervalo de confianza de $95 \%$.

CUADRO 5. Costos medios (en US\$ por caso) del tratamiento de corta duración bajo observación directa (DOTS) y del DOTS residencial (DOTS-R) para pacientes con tuberculosis pulmonar, según la fuente de pago, 2010, Medellín, Colombia

\begin{tabular}{lcccc}
\hline \multicolumn{1}{c}{ Fuente } & DOTS & $\%$ & DOTS-R & $\%$ \\
\hline Sistema de salud & 163,9 & 21,0 & 163,9 & 19,2 \\
Pacientes y familiares & 403,8 & 51,8 & 412,5 & 48,2 \\
Secretaría de Salud & 212,3 & 27,2 & 278,9 & 32,6 \\
Costo medio total & 780,0 & 100,0 & 855,3 & 100,0 \\
\hline
\end{tabular}

Fuente: elaboración de los autores.

Nota: Las cifras fueron estimadas a partir del tipo de cambio vigente en diciembre de 2010 (US\$1 = \$1914).

CUADRO 6. Razones de costo-efectividad y costos incrementales del tratamiento de corta duración bajo observación directa (DOTS) y del DOTS residencial (DOTS-R) para pacientes con tuberculosis pulmonar, usando un grupo de comparación del mismo año (2006) y otro de dos períodos distintos (2006 y 2009), Medellín, Colombia

\begin{tabular}{|c|c|c|c|c|}
\hline \multirow[b]{2}{*}{ Razón } & \multicolumn{2}{|c|}{ DOTS } & \multicolumn{2}{|c|}{ DOTS-R ${ }^{a}$} \\
\hline & Curados & Éxitos ${ }^{b}$ & Curados & Éxitos \\
\hline \multicolumn{5}{|c|}{ Pacientes diagnosticados el mismo año (2006) } \\
\hline Costos del tratamiento (US\$) & 78000,0 & 77934,2 & 85530,0 & 85474,5 \\
\hline Resultados del tratamiento (No.) & 68,6 & 80,0 & 76,2 & 87,8 \\
\hline Costo-efectividad (US\$ por caso) & 1137,0 & 974,2 & 1122,4 & 973,5 \\
\hline Cambio en los costos (US\$) & $\ldots{ }^{c}$ & $\ldots$ & 7530,0 & 7540,3 \\
\hline Cambio en los resultados (No.) & $\ldots$ & $\ldots$ & 7,6 & 7,8 \\
\hline Costo incremental (US\$) & $\ldots$ & $\ldots$ & 990,8 & 966,4 \\
\hline \multicolumn{5}{|c|}{$\begin{array}{l}\text { Pacientes diagnosticados en dos períodos distintos } \\
\text { (2006 y 2009) }\end{array}$} \\
\hline Costos del tratamiento (US\$) & 78000,0 & 77890,1 & 85530,0 & 85474,5 \\
\hline Resultados del tratamiento (No.) & 52,2 & 66,1 & 74,2 & 85,5 \\
\hline Costo-efectividad (US\$ por caso) & 1494,3 & 1178,4 & 1152,7 & 999,7 \\
\hline Cambio en los costos (US\$) & $\ldots$ & $\ldots$ & 7530,0 & 7540,3 \\
\hline Cambio en los resultados (No.) & $\ldots$ & $\ldots$ & 22,0 & 19,4 \\
\hline Costo incremental (US\$) & $\ldots$ & $\ldots$ & 342,3 & 388,7 \\
\hline
\end{tabular}

Fuente: elaboración de los autores.

Nota: Las cifras fueron estimadas a partir del tipo de cambio vigente en diciembre de 2010 (US\$1 = \$1914).

a Se usó una cohorte de 100 pacientes como grupo de comparación.

${ }^{\mathrm{b}}$ Estos resultados incluyen curados y tratamientos terminados.

${ }^{c}(\ldots): \sin$ datos.

su mayor efectividad se relaciona en gran medida con las actividades de educación en salud llevadas a cabo en los hogares; con la estabilidad y capacidad técnica del equipo de salud encargado de hacer el seguimiento, y con el abordaje integral del paciente y su familia en su lugar de residencia.
Si bien la intervención evaluada en el presente trabajo no es exactamente igual a ninguna de las variantes del DOTS centradas en el paciente que han sido publicadas hasta el momento, los resultados son comparables a los obtenidos por Prado y colaboradores en Brasil (24). Este estudio comparó un DOTS tradicional, en el cual personal de salud para apoyo comunitario suministraba el tratamiento desplazándose hacia donde estuviese el paciente, con otro enfoque más centrado en el núcleo familiar, donde el tratamiento era suministrado por un guardián elegido entre los integrantes del núcleo familiar, quien recibía entrenamiento. Esta alternativa también promueve y ofrece educación en salud a los pacientes y a su grupo familiar. Tanto el estudio de Brasil como el presente, encuentran que la supervisión domiciliaria con apoyo de miembros del grupo familiar resulta más efectiva y menos costosa que la tradicional. Khan y colaboradores también informan resultados en el mismo sentido, donde el DOTS proporcionado en los centros de salud registró los peores resultados en costo-efectividad cuando se lo compara con alternativas centradas en el paciente y su entorno (30).

Varios de los estudios sobre DOTS llevados a cabo durante la última década documentan en parte lo mencionado anteriormente, en el sentido de que las mejores alternativas de implementación del DOTS consisten en gestionar el tratamiento con acciones por fuera del ámbito hospitalario, como un modo de involucrar a otros miembros de la comunidad o del hogar y desarrollar con ellos educación primaria en salud (23, 31-34). Entre otros trabajos que aplicaron esta perspectiva, cabe mencionar el de Nganda, en Kenya (21), y el de Floyd, en Malawi (22), los cuales reportan sensibles reducciones de costos con la implementación de un DOTS ambulatorio, en el marco de alianzas público-privadas que incluían capacitación intensificada al personal de salud.

El presente estudio, sin embargo, presenta algunas limitaciones que conviene hacer explícitas. La primera tiene que ver con haber asumido parcialmente un enfoque de costeo de arriba-abajo para el manejo del programa por parte de la Secretaría de Salud y para el tratamiento que se dispensa a los pacientes, pues se trata de información secundaria que podría estar conllevando grandes ineficiencias en la gestión de estas instituciones. En el caso del costeo del tratamiento, 
este sesgo pudo ser menos relevante en la medida en que la fuente de información era más representativa porque incorporaba un número importante de instituciones prestadoras de servicios de salud públicas y privadas.

Una segunda limitación se relaciona con la calidad de la información por parte del programa de control y de las entidades participantes. Los datos usados en los dos sets de grupos de comparación establecidos (dos grupos en 2006 y dos grupos [antes-después] en 2006 y 2009) se conformaron con base en los registros institucionales del programa municipal de control de tuberculosis, los cuales, aun cuando han venido mejorando en su calidad, todavía son incompletos. Adicionalmente, los criterios de selección del grupo intervenido se establecieron para población mayor de 15 años que pudiera ser seguida en el tiempo por al menos dos años, lo que ponía en condiciones favorables a los pacientes del grupo intervenido en comparación con los pacientes del programa tradicional. En este caso, sin embargo, luego de ajustarse las diferencias entre los grupos en el análisis, las conclusiones se mantuvieron.

Por otra parte, los diferenciales en efectividad a favor del DOTS-R podrían estar relacionados con la mayor propor- ción de afiliados al régimen contributivo en salud observada en este grupo de pacientes, que contrasta con la mayor proporción de pacientes inscritos en el régimen subsidiado pertenecientes al grupo de DOTS convencional. Aun así, no es en el régimen subsidiado donde se presentan las mayores barreras de acceso a los servicios de salud. Se ha demostrado que en la ciudad de Medellín, cerca de $80 \%$ de las tutelas en salud por negación de servicios se presentan en el régimen contributivo (35). Además, los pacientes del régimen subsidiado acuden con mayor frecuencia a la red pública, donde hay más tradición en el manejo programático de la TB.

El presente estudio documentó la magnitud con la que el DOTS-R impacta en los costos que debe sufragar la Secretaría de Salud. En este punto, es importante tomar en consideración que dichos costos se derivan del uso de recursos logísticos y de personal especializado, que podrían ser ahorrados mediante economías de escala en el caso de que esta entidad asumiese las tareas de seguimiento a los hogares de los pacientes. De hecho, la Secretaría de Salud tiene un amplio potencial para mejorar la productividad de sus servicios, pues algunas de las acciones que por ley debe desarrollar son similares a las asumidas en la labor de seguimiento a pacientes y sus familiares, y su articulación bien podría dar soporte a la adopción institucional del DOTS-R.

Finalmente, con base en los resultados obtenidos, se recomienda a las autoridades del sector salud de Medellín que incorporen al programa de control de tuberculosis acciones de seguimiento y acompañamiento a pacientes e integrantes de su núcleo familiar. La adición de un componente de DOTS-R, sin embargo, deberá ser respaldada por el personal de salud y los recursos físicos y financieros que apoyan actualmente al programa de control de TB, y deberá ser evaluada detallada y cuidadosamente. Dado que el DOTS-R aquí descrito constituye un agregado relativamente sencillo al DOTS convencional, sería conveniente realizar más evaluaciones del DOTS-R en otros sitios endémicos, donde todavía se esté realizando el DOTS centrado en los puestos de salud.

Agradecimiento. Los autores desean expresar su gratitud por el respaldo financiero brindado por las siguientes instituciones: Colciencias; Universidad de Antioquia, Facultad Nacional de Salud Pública; Instituto Metropolitano de Salud de Medellín, y la Secretaría de Salud Municipal (Medellín).

\section{REFERENCIAS}

1. Organización Mundial de la Salud, Tuberculosis, Nota descriptiva $N^{\circ} 104$, marzo de 2012. Disponible en: http://www.who.int/ mediacentre/factsheets/fs104/es/index.html Acceso el 26 de agosto de 2012.

2. World Health Organization. The global burden of disease: 2004 update. Disponible en: http:/ / www.who.int/healthinfo/global_burden disease /2004_report_update/en/index. $\mathrm{html}$ Acceso el 03 de septiembre de 2012.

3. Pan American Health Organization. Regional Plan for Tuberculosis Control, 2006-2015. Washington, D.C.: PAHO; 2006.

4. Organización Panamericana de la Salud. Salud en las Américas 2007. Vol. I. Washington, D.C.: OPS; 2007.

5. Smith I. ¿Cuál es la carga económica, social y sanitaria de la tuberculosis? Tuberculosis: detección de casos, tratamiento y vigilancia: preguntas y respuestas. 2. ${ }^{a}$ ed. Washington, DC: Organización Panamericana de la Salud; 2006.

6. Gold MR, Siegel JE, Russell LB, Weinstein MC. Cost-Effectiveness in Health and Medicine. New York: Oxford University Press; 1996.
7. Floyd K, Wilkinson D, Gilks C. Comparison of cost effectiveness of directly observed treatment (DOT) and conventionally delivered treatment for tuberculosis: experience from rural South Africa. BMJ. 1997;315(7120): 1407-11.

8. Murray CJ, DeJonghe E, Chum HJ, Nyangulu DS, Salomao A, Styblo K. Cost effectiveness of chemotherapy for pulmonary tuberculosis in three sub-Saharan African countries. The Lancet. 1991;338(8778):1305-8.

9. World Health Organization. The Stop TB strategy: Building on and enhancing DOTS to meet the TB-related Millennium Development Goals. Geneva: WHO; 2006.

10. Volmink J, Garner P. Directly observed therapy for treating tuberculosis. Cochrane Database Syst Rev. 2006(2):CD003343.

11. Simmon M, Rubinstein F. Controversia sobre la efectividad del tratamiento supervisado en tuberculosis. Evid Actual Pract Ambul. 2007;10(1):23-5.

12. Smith I. ¿Es el DOTS eficaz en función de los costos? Tuberculosis: detección de casos, tratamiento y vigilancia: preguntas y respues- tas. 2. ${ }^{a}$ ed. Washington, DC: Organización Panamericana de la Salud; 2006.

13. Burman WJ, Dalton CB, Cohn DL, Butler JR, Reves RR. A cost-effectiveness analysis of directly observed therapy vs self-administered therapy for treatment of tuberculosis. Chest. 1997;112(1):63-70.

14. Snyder DC, Chin DP. Cost-effectiveness analysis of directly observed therapy for patients with tuberculosis at low risk for treatment default. Am J Respir Crit Care. 1999;160(2): 582.

15. Vassall A, Bagdadi S, Bashour H, Zaher H, Maaren P. Cost-effectiveness of different treatment strategies for tuberculosis in Egypt and Syria. Int J Tuberc Lung Dis. 2002;6(12): 1083-90.

16. Mohan CI, Bishai D, Cavalcante S, Chaisson RE. The cost-effectiveness of DOTS in urban Brazil. Int J Tuberc Lung Dis. 2007;11(1): 27-32.

17. Steffen R, Menzies D, Oxlade O, Pinto M, de Castro AZ, Monteiro P, et al. Patients' Costs and Cost-Effectiveness of Tuberculosis Treatment in DOTS and Non-DOTS Facilities in 
Rio de Janeiro, Brazil. PloS One. 2010;5(11): e14014.

18. World Health Organization. Guidelines for cost and cost-effectiveness analysis of tuberculosis control. Geneva: WHO; 2002.

19. Drummond MF, O’Brien BJ, Stoddart GL, Torrance GW. Métodos para la evaluación económica de los programas de asistencia sanitaria. 2. ${ }^{a}$ ed. Madrid: Díaz de Santos; 2001.

20. Pinto JL, Sánchez FI. Métodos para la evaluación económica de nuevas prestaciones. Barcelona: Centre de Recerca en Economia I Salut/ Ministerio de Sanidad y Consumo; 2004.

21. Nganda B, WangOombe J, Floyd K, Kangangi J. Cost and cost-effectiveness of increased community and primary care facility involvement in tuberculosis care in Machakos District, Kenya. Int J Tuberc Lung Dis. 2003; 7(9s1):S14-20.

22. Floyd K, Skeva J, Nyirenda T, Gausi F, Salaniponi F. Cost and cost-effectiveness of increased community and primary care facility involvement in tuberculosis care in Lilongwe District, Malawi. Int J Tuberc Lung Dis. 2003;7(9s1):S29-37.

23. Floyd K, Arora VK, Murthy KJ, Lonnroth K, Singla N, Akbar Y, et al. Cost and costeffectiveness of PPM-DOTS for tuberculosis control: evidence from India. Bull World Health Organ. 2006;84(6):437-45.

24. Prado TN, Wada N, Guidoni LM, Golub JE, Dietze R, Maciel EL. Cost-effectiveness of community health worker versus homebased guardians for directly observed treatment of tuberculosis in Vitória, Espírito Santo State, Brazil. Cad Saude Publ. 2011;27(5): 944-52.

25. del Corral H, París SC, Marín ND, Marín DM, López L, Henao HM, et al. IFNy Response to Mycobacterium tuberculosis, Risk of Infection and Disease in Household Contacts of Tuberculosis Patients in Colombia. PLoS One. 2009:4(12):e8257.

26. Ministerio de Salud y la Protección Social, Colombia. Guía de atención de la tuberculosis pulmonar y extrapulmonar. Colombia: Ministerio de Salud y la Protección Social; 2007.

27. Alcaldía de Medellín, Colombia. Encuesta de calidad de vida en Medellín, 2009. Medellín: Departamento Administrativo de Planeación; 2010.

28. Fletcher RH, Fletcher SW, Wagner EH. Treatment. Clinical Epidemiology: The Essentials. 3. ${ }^{\text {a }}$ ed. Baltimore: Williams \& Wilkins; 1996.

29. Secretaría Municipal de Salud de Medellín, Colombia. Estadísticas de los casos diagnosticados con tuberculosis, 2006-2009. Medellín: Secretaría Municipal de Salud de Medellín; 2011.

30. Khan MA, Walley JD, Witter SN, Imran A, Safdar N. Costs and cost-effectiveness of different DOT strategies for the treatment of tuberculosis in Pakistan. Health Policy Plan. 2002;17(2):178.
31. Wyss K, Kilima P, Lorenz N. Costs of tuberculosis for households and health care providers in Dar es Salaam, Tanzania. Trop Med Int Health. 2001;6(1):60-8.

32. Sinanovic E, Kumaranayake L. Financing and cost-effectiveness analysis of public-private partnerships: provision of tuberculosis treatment in South Africa. Cost Eff Resour Alloc. 2006;4(1):11.

33. Islam MA, Wakai S, Ishikawa N, Chowdhury AM, Vaughan JP. Cost-effectiveness of community health workers in tuberculosis control in Bangladesh. Bull World Health Organ. 2002;80(6):445-50.

34. Martínez-Medina MA. Efecto del tratamiento acortado estrictamente supervisado (TAES) sobre la adherencia y la tasa de curación de la tuberculosis pulmonar. Gac Med Mex. 2004;140(1):1-6.

35. Nieto E, Arango CA. Costos de los servicios de salud tutelados y del proceso legal de las tutelas en Medellín, 2009. Rev Fac Nac Salud Publica. 2011;29(3):223-31.

Manuscrito recibido el 18 de octubre de 2011. Aceptado para producción, tras revisión, el 10 de julio de 2012.
ABSTRACT

Cost-effectiveness of an
alternative tuberculosis
treatment: home-based
guardian monitoring of
patients

Key words
Objective. Estimate the cost-effectiveness ratio of the directly observed treatment short course (DOTS) for treatment of tuberculosis (TB), comparing it to a variation of this treatment that includes increased home-based guardian monitoring of patients (DOTS-R).

Methods. Taking a social perspective that includes the costs for the health institutions, the patients, and their family members, and for other entities that contribute to making operation of the program effective, the costs incurred with each of the two strategies were evaluated and the cost-effectiveness ratios were estimated adopting the measures of effect used by the control programs. The estimate of the cost of each of the two strategies includes the cost to the health institutions that administer treatment, the patients and their family members, and the cost to the Ministry of Health that manages public health programs on the municipal level. Based on these costs and the number of cases cured and treatments completed as outcome measures of each of the strategies evaluated, the cost-effectiveness ratio and incremental cost were calculated.

Results. The DOTS-R was found to be more cost-effective for achievement of successful treatments than the DOTS. The DOTS-R recorded costs of US\$ 1122.40 to US\$ 1152.70 for each case cured compared to values of US\$ 1137.00 to US\$ 1494.30 for the DOTS. The percentage of cases treated successfully was higher with DOTS-R than with DOTS.

Conclusions. The DOTS-R is a promising cost-effective alternative for improved control of TB in endemic areas. It is recommended that the health authorities include home-based guardian monitoring of patients in their institutional management of the TB program, with the participation of health workers and the physical and financial resources that currently support this program.

Tuberculosis, pulmonary; cost-efficiency analysis; financing; health; Colombia. 